\title{
TYPES, PAPER AND PRINTING IN RELATION TO EYESTRAIN
}

BY

\section{R. B. Fishenden}

Comport in reading is as important to those concerned in producing books as to the ophthalmic surgeon. Considerable work has been done on the subject by, workers who have approached the subject scientifically and by those who have made type legibitity a special study. Unfortunately these investigations do not appear to have been correlated, even in recent times, although instruments have been devised to measure complex " reader reactions" : there are many diverse factors to be taken into account and these may lead to inaccurate conclusions if the approach is not sound.

In considering briefly some points of interest a few assumptions will be made. Illumination will be on the basis of bright diffused daylight or artificial illumination, say twenty foot-candles. The papers used are either white or lightly tinted, and the printing done in black ink.

In an early investigation on types for school books ${ }^{1}$ sound conclusions were reached. The report was against condensed types, or narrow breadth being more important than height. It was recognised that contrast between fine and heavy strokes should be avoided. De Vinne, referring to this subject, said that "the punch cutter straining after a hair line that just stops before invisibility is ably seconded by the pressman who scantily inks these light faces."

Apart from dimensions of the letters, fine hair lines are likely to approach the visual threshold, as are serifs-although these, if well designed, are an aid to differences in letter forms and thus to legibility. It is evident that types which do not exhibit an extreme of contrast between thick and thin strokes will be more restful. The difference between these two groups of type design is here illustrated.

\section{In every period there have $b$ In every period there have been}

FIG. 1.

The first example shows normal contrast between thick and thin lines. the second excessive contrast. The second shows the thick lines arranged vertically, while the first shows them at an angle which aids the flow of easy reading. 
Well designed sans serif types have their value, as is demonstrated in the Edward Johnston poster type for London Underground and Gill Sans by Eric Gill.

An early investigator, Legros, ${ }^{2}$ showed that there is a greater coincidence between the characters in the alphabets of some type forms. This is of more importance in figures than in letters; differences between two designs of figures are shown.

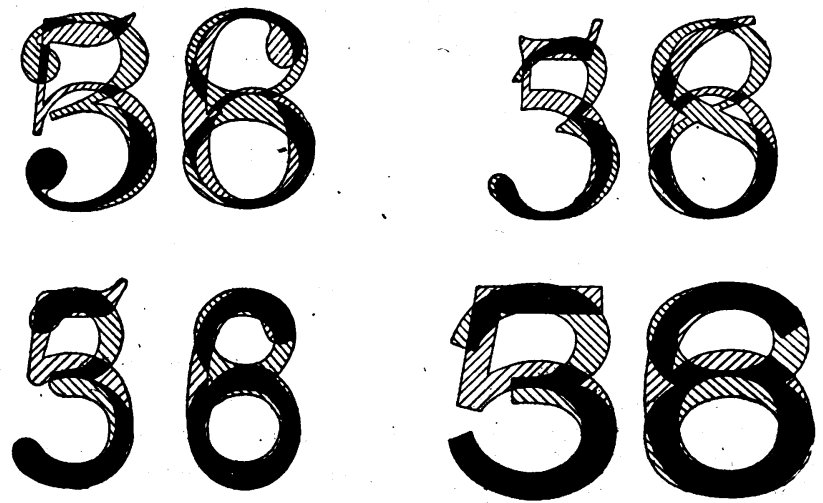

FIG. 2.

It is sometimes considered that legibility is aided, particularly in figures, by maximum differences. The diagram shows four series, the the solid black showing the co-incidences. From this aspect the first is the best and the last the least successful.

In the design of types there should be no serious departure from accustomed shapes. The types for timetables have been specially studied and figures designed to provide maximum differences in outline without departing from the recognised forms. The legibility of both figures and letters is dealt with in a report on Government Printing, ${ }^{3}$ which advised that " types in

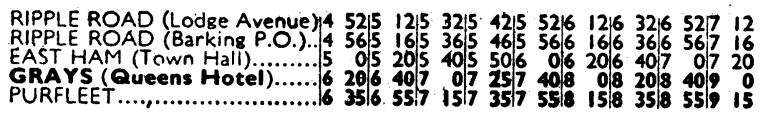

FIG. 3 .

Time table figures are specially designed for maximum legibility. This example is regarded as one of the most successful.

which the contrast between the thick and thin strokes is exaggerated, or in which the face is unduly compressed, should not bc used." A section of timetable figures of London Transport i: here shown. 
Apart from configuration, the major factor in type legibility is size. Types nowadays are known by their point size, there being seventy-two points to the inch. This article is set in 11 point old style type and the heading in antique old style, a heavy type of similar character. It is usually considered sufficient to a type in which the metal is one-sixth of an inch in depth. Legibility depends on the size of the small or lower case letters, known as " $x$ " height, and although this is well understood there are frequent misunderstandings even in official instructions. Differences in $\mathrm{x}$ height of the small letters in type series are shown

7 point Ionic 451 (soLID)
In every period there have
been better or worse types
employed in better or
worse ways. The better
types employed in better

8 point Times 327 (SOLID)

In -every period there have been better or worse types employed in better or worse ways. The better types employed in better ways have

\begin{abstract}
7 point Ionic (LEADED 3 pts.)
In every period there have

been better or worse types

employed in better or

worse ways. The better
\end{abstract}

8 point Times 327 (LEaded 2 PTS.)

In every period there have

been better or worse types

employed in better or worse

ways. The better types em-

FIG. 4.

Examples on the left with the types unleaded show that the size is not related to the size of face, the smaller 7-point "Ionic" being larger than the 8-point "Times." On the right the two types have leads between the lines which markedly increases legibility.

in the following examples on the left; although " Ionic", is on a smaller body, the face is larger than " Times."

Legibility will be reduced if the letters of words are packed too closely. The spacing between words is also important; if too close, reading is made more difficult and if too wide the appearance of the page is not so good.

The reading reactions to wide spacing between lines have been measured. Legibility is improved by inserting a space between the lines of type, called "leading," and it is a corrective for too long lines (see examples above on the right). Increased leading naturally increases the number of pages. The length of line requires to be related to the type size.

There are notable differences in legibility in the design of types, a subject which has been discussed by Morison, ${ }^{4}$ Warde, ${ }^{5}$ Tarr $^{6}$ and others. '

Times Roman is a successful modern type whose legibility is shown to advantage in small advertisements. 
10 point Fournier 185 (solid)

In every period there have been better or worse types, employed in better or: worse ways. The better types employed in better ways have been used by the educated printer acquainted with standards and history, directed

12. point Baskerville 169

In every period there have been better or worse types, employed in better or worse ways. The better types employed in better

\begin{abstract}
12 point Times 327
In every period there have been better or worse types, employed in better or worse ways. The better types employed in better

12 point Walbaum 374

In every period there have been better or worse types, employed in better or worse ways. The better types employed in better
\end{abstract}

FIG. 5.

These are four types, all of good design, widely used. Baskervilleiisia popular type for books, also Fournier and Walbaum. "Times" may be the best type for newspaper printing, and is now widely used for a variety of purposes.

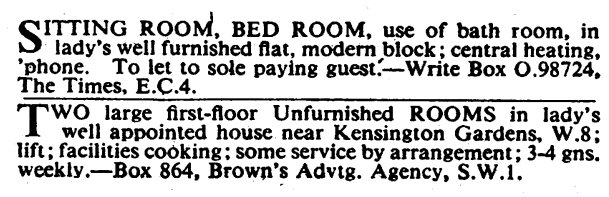

FIG. 6.

Small advertisements in newspapers are an extreme test for legibility. These two advertisements are set in "Times" and show unusual clarity.

Visual acuity is stated to be in the order of one minute of arc in resolving two adjacent black lines. In an illustration by the halftone process of coarse screen, as for example a newspaper halftone or a half-tone poster, the angle at which the dots fail to be resolved is less than half a minute. ${ }^{7}$ Experiment shows there is a sharp end point in the visual reaction when the individual halftone dots cannot be resolved. ${ }^{8}$

The design of type for book work embraces aesthetic consideration as well as the more mundane aspects of visibility, and this 
has sometimes been overlooked in investigations. 9 A well designed book type assists the eye to flow along the line and there is a marked psychological difference between such a type and one which has the effect of halting the eye at each word,-although the type may be legible. A happy example of a good type of this kind is here shown in contrast with a poor one. Idiosyncrasies in, or unfamiliar forms of letters interrupt smooth reading.

The influence of paper on appearance, legibility and the reader's comfort needs consideration. Smoothness, relative hardness of the surface, opacity, coating and gloss, are the chief factors. To ensure that a type shall print with clarity, a smooth

\title{
12 point Mediaeval 136 . \\ In every period there have been better or worse types, employed in better or worse ways. The better types employed in better
}

\author{
14 point Bembo 270 \\ In every period there have been better or \\ worse types, employed in better or worse \\ ways. The better types employed in better
}

FIG. 7.

\begin{abstract}
The second example, "Bembo," has some similarity to the first example in Fig. 1. The type is well proportioned and the long descending characters are helpful and in contrast with the example of "Times" in Fig. 5. The absence of disturbing elements and the oblique stress help easy reading. "Mediaeval" is a bad type from the standpoint of legibility; the unusual shape of some of the letters causes the eye sub-consciously to halt.
\end{abstract}

paper, white or off-white, is preferred. A rough paper known as an " antique " or a " featherweight," sometimes used by publishers to give novels a deceptive bulk, needs more ink and pressure on the printing machine. The first effect of this rough paper is that the extra pressure and ink cause the types to print more heavily than smooth ones.

In the early days of printing damped paper was used, which enabled results to be produced with less pressure than would be necessary on a dry paper. In these conditions the types printed more heavily and it is important to remember that the types were designed for this kind of treatment; if these types are printed on a smooth paper they tend to give a spidery appearance.

One of the drawbacks of the heavy inking and pressure necessary for printing dry " antique" paper is that with small types, and even book types, there is a tendency for the type to fill in ; this applies particularly in such letters as the loop of the small 
" $\mathrm{e}$ " and some types are designed to reduce this tendency to a minimum. Line illustrations, particularly those with fine detail, print better on a smooth paper.

Apart from the smoothness of a paper surface, a relatively hard one-the hardness depending on the fibres used and on the sizing -needs more pressure on the printing machine than does a soft one : a nice balance is required between the right fibre, the sizing and the surface, to ensure good printing and minimum trouble for the printer. If the right paper is chosen, half-tone illustrations with a medium fine screen, say 120 lines to the inch, can be printed adequately, and in some cases a finer screen may be used; a good example of a paper of this kind was used in the British Journal of Ophthalmology prior to the war.

With the introduction of paper restrictions and the need for the greatest economy, papers of light substance have been used for book work and in some cases these have lacked the required opacity. Opacity and substance are not directly related because the treatment of the fibres in making the paper has some influence, and it is also possible to add loading material to suitable papers to increase opacity. When using a paper of low opacity eyestrain may be induced because the ink on the reverse side, and also on the next sheet, becomes apparent. This effect is known as " show through." In papers of this class the nature of the ink should be arranged so that the medium, or liquid part of the ink, does not penetrate the paper unduly. Where the ink is defective in this respect the effect is bad and the phenomenon is known as " strike through." Papers used for printing Bibles are of thin substance but they have a relatively high opacity.

Art paper comprises a body paper coated with china clay, usually bound in casein, and the paper is calendered to give it a high gloss. Papers of this kind were evolved to render adequately the detail in blocks made by the half-tone process. On uncoated papers the individual dots may be in some measure distorted, which causes the details to be poorly rendered. Such is the justification for using art papers, and they give the maximum contrast between the blackness of the ink and the whiteness of the paper coating. Apart from these technical considerations, glossy coated papers are best avoided because they are tiring for the reader.

A smooth uncoated paper has a good diffuse reflection so that whatever the angle of incidence of the light may be, there is little or no glare. Owing to its high gloss, the art paper has a high specular reflection and the tiring glare accompanying these papers will be recognised. Papers free from glare which would print half-tone blocks reasonably well were available before the war and it is felt that these papers have value on technical, physiological and aesthetic grounds. 
Given a type of high legibility, and paper of a suitable kind, the quality of the printing has a marked influence on the effect of the printed pages and on the readability. Uneven and bad inking induces eye-strain. The printability of the paper and the care exercised by the printer have a marked effect on the clarity of illustrations. ${ }^{10}$

These brief notes on paper refer to letterpress printing and the subject requires study in different ways when the lithographic offset and photogravure processes are used.

\title{
- REFERENCES
}

1. Report of the influence of school books on eyesight. British Association for Advancement of Science, 1912.

2. Typographical Printing Surfaces. Grant and Legros. 1916, p. 160.

3. Report on Type for Government Printing.' H.M.S.O., 1922.

4. First Principles of Typography, Morison. Fleuron, 1930, Vol. VII, p. 55.

5. Size of Print, Warde. Penrose Annual, 1938, Vol. XL, p. 75.

6. Printing To-day, Tarr, p. 70.

7. Physiologic Optics, Tscherning, 1900, Chapter XVIII.

8. Half-tone Posters, Fishenden. Penrose Annual, 1911, Vol. XVII, p. 185.

9. Reading as a Visual Task, Luckeish and Moss, 1942, Chapter VI.

10. The Printability of Paper, Poulter and Croney, Paper Makers' Association, Great Britain, 1941.

\section{OPHTHALMIC TREATMENT IN THE FIELD, 1943*}

BY

\author{
G. C. Dansey-Browning, Major, R.A.M.C. \\ "The Cyclops, a people of Sicily ...." The Odyssey
}

THE object of this article is to make a report on the present medical state of the ophthalmic battle-casualties from the Sicily and early Italian campaigns. My reason is the remark heard a long time ago of a cynic who left the operating theatre muttering" "Very neat, but I would like to see that eye in two years time!"

\section{The mobile ophthalmic unit}

The conditions under which the unit worked were scarcely orthodox. Let me attempt to set the scene for you!

Take a case of instruments, a packful of test-types, loupes, the ophthalmoscope, and grab the first hospital-ship available. Your last-minute choice of essentials are flung at your orderly, together with the spare pair of shorts and shirt that will constitute ail your "others" for the next three months.

Berth in Syracuse harbour for a hair-raising spell between a

* Received for publication, August 28, 1945. 